\title{
Statistical 3D ‘atomistic’ simulation of decanano MOSFETs
}

\author{
A. Asenov ${ }^{\dagger}$, G. Slavcheva, A. R. Brown, R. Balasubramaniam, \\ J. H. DAVIES \\ Device Modelling Group, Department of Electronics \& Electrical Engineering, University of Glasgow, \\ Glasgow G12 8LT, U.K.
}

(Received 9 November 1999)

\begin{abstract}
A 3D statistical 'atomistic' simulation technique has been developed to study the effect of the random dopant induced parameter fluctuations in aggressively scaled MOSFETs. Efficient implementation of the 'atomistic' simulation approach has been used to investigate the threshold voltage standard deviation and lowering in the case of uniformly doped MOSFETs, and in fluctuation-resistant architectures utilising epitaxial-layers and delta-doping. The effect of the random doping in the polysilicon gate on the threshold voltage fluctuations has also been thoroughly investigated. The influence of a single-charge trapping on the channel conductivity in decanano MOSFETs is studied in the 'atomistic' framework as well. Quantum effects are taken into consideration in our 'atomistic' simulations using the density gradient formalism.
\end{abstract}

(C) 2000 Academic Press

Key words: MOSFET, doping, fluctuations, semiconductor device simulation, threshold, ultra-small devices, quantum effects.

\section{Introduction}

When scaling device dimensions down to the deep submicron range the particular microscopic nature of the random discrete dopant charge distribution in the channel region becomes of great significance to device performance and operation [1-3]. The simulation of a single device with continuous charge distribution is unable to reproduce the experimentally observed statistical fluctuations in device characteristics [4-12]. Therefore a statistical approach to the simulations is required in order to account for the various microscopically different random charge distributions in macroscopically identical devices. This approach implies an estimation of the basic design parameters (such as threshold voltage, subthreshold slope, transconductance, driving current, etc.) averaged over the statistical ensemble of microscopically different devices, rather than predicting the characteristics of a single device with continuous doping. In order for the charge of each individual dopant to be spatially resolved, full-scale 3D atomistic simulations with fine grain discretization are required [13]. Thus, the statistical atomistic simulations represent essentially a 4D problem, the fourth dimension being the size of the statistical ensemble.

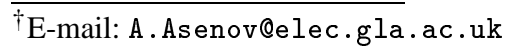




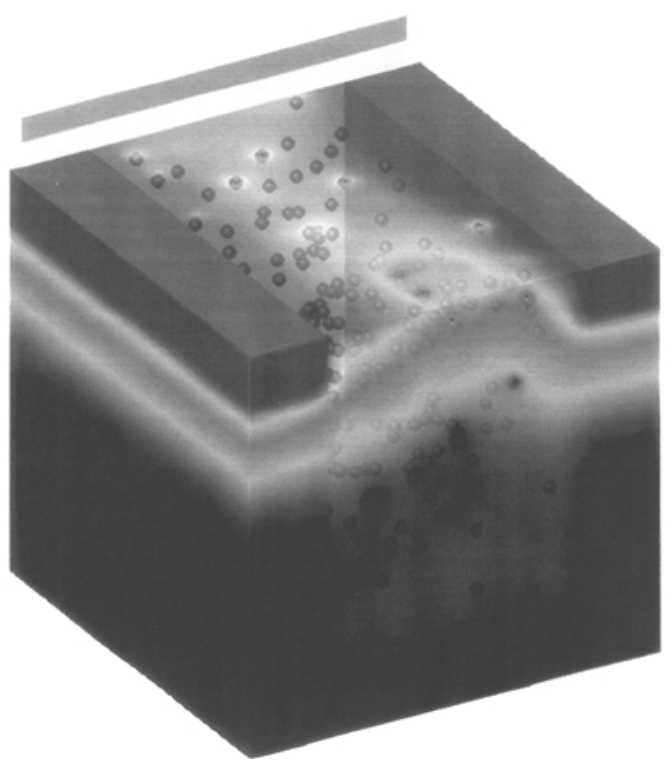

Fig. 1. Typical solution domain for atomistic simulations showing the location of individual dopants in a $30 \times 50 \mathrm{~nm}^{2} \mathrm{MOSFET}$, with the potential distribution obtained from the solution of Poisson's equation superimposed on top.

\section{Classical atomistic simulations}

In many cases, in order to investigate the effects of random dopant induced fluctuations it is sufficient to perform a classical simulation involving the solution of the drift-diffusion approximation to the Boltzmann transport equation. This has been the basis for the majority of the work performed so far in this field $[14,15]$. The standard drift-diffusion approach requires the solution of Poisson's equation along with the current continuity equations for electrons, holes or both. The drift-diffusion simulator can be used to perform statistical investigations into threshold voltage fluctuations and lowering in MOSFETs scaled below $0.1 \mu \mathrm{m}$ under arbitrary bias conditions. Short-channel effects such as drain-induced barrier lowering (DIBL) are naturally included in this approach, together with asymmetry in device characteristics due to dopant clustering at one end of the channel.

We have developed a 3D 'atomistic' drift-diffusion simulator based on the decoupled Gummel procedure [16]. The solution of Poisson's equation uses a parallelized black/red Newton SOR solver, while a parallelized BiCGSTAB solver with polynomial preconditioning has been implemented for the solution of the electron current continuity equation. A typical solution domain used in our simulator is shown in Fig. 1 showing the location of random dopants in a $30 \times 50 \mathrm{~nm}^{2}$ MOSFET, with the potential distribution superimposed on top. The discrete dopants are placed in the outlined channel region between the source and drain. In the rest of the simulation domain, the doping charge has a continuous distribution. The expected number of dopants in the atomistic region is estimated by integration of the continuous doping distribution. The actual number of dopants in each MOSFET from the simulated sample is chosen randomly from a Poisson distribution with this mean. Then the dopants are placed randomly according to the initial continuous doping distribution using a rejection technique.

While it is possible to perform statistical analysis using the fully self-consistent solution, the time and memory requirements, particularly for the solution of the current continuity equation, make this a less favourable option when a large ensemble of devices are to be simulated. If the MOSFET is properly scaled and does not exhibit DIBL then it is possible to reduce the computational and memory burden by solving 


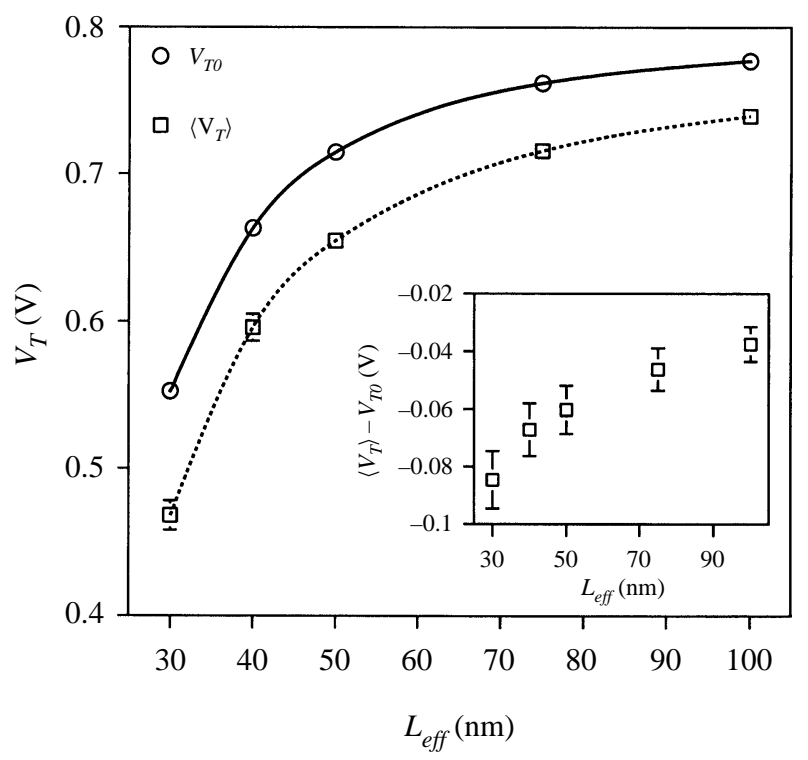

Fig. 2. Comparison of 'atomistically' simulated average threshold voltage $\left\langle V_{T}\right\rangle$ and $V_{T 0}$ for devices with continuous doping for a set of MOSFETs with different channel lengths. $W_{\text {eff }}=50 \mathrm{~nm}, N_{A}=5 \times 10^{18} \mathrm{~cm}^{-3}$, and $t_{o x}=3 \mathrm{~nm}$. Samples of 200 transistors.

the current continuity equation only in a thin slab extending from the interface to a depth $d$, much smaller than the total depth of the solution domain [17]. In a properly scaled $50 \mathrm{~nm}$ MOSFET, a slab of only $1 \mathrm{~nm}$ produces an error of less than $3 \%$ in the current calculated. This error can be reduced below $0.1 \%$ if a $3 \mathrm{~nm}$ slab is used.

At low drain voltages a further simplification to the simulation methodology is possible, which allows rapid, accurate calculation of current and threshold voltages [15]. The solution of the nonlinear Poisson equation is obtained for a particular gate voltage, $V_{G}$, for a zero-potential difference between the source and the drain. The carrier concentration obtained from this solution is used for calculating the resistance of the device and hence the current.

\section{Applications}

\subsection{Uniformly doped devices}

The use of discrete doping distributions in the simulations results not only in fluctuations in the MOSFET parameters, but also in average values different from the results of the continuous charge simulations. For example, dopant discreteness and randomness introduces not only fluctuations but also lowering in the threshold voltage compared with the continuous charge simulations. In Fig. 2 the effective channel length dependence of the average threshold voltage, $\left\langle V_{T}\right\rangle$, from atomistic simulations, and threshold voltage from continuous charge simulations, $V_{T 0}$, are compared for transistors with $W_{e f f}=50 \mathrm{~nm}, N_{A}=5 \times 10^{18} \mathrm{~cm}^{-3}$ and $t_{o x}=3 \mathrm{~nm}$. The inset in Fig. 2 shows that the random dopant induced threshold voltage lowering is channel length dependent and increases rapidly below a $50 \mathrm{~nm}$ effective channel length, reaching almost $0.1 \mathrm{~V}$ in a $30 \mathrm{~nm}$ MOSFET.

The atomistically calculated doping concentration dependence of the threshold voltage standard deviation in the sub-100 nm channel length MOSFETs is stronger $\left(N_{A}^{0.40}\right)$ than the $N_{A}^{1 / 4}$ dependence adopted in most 


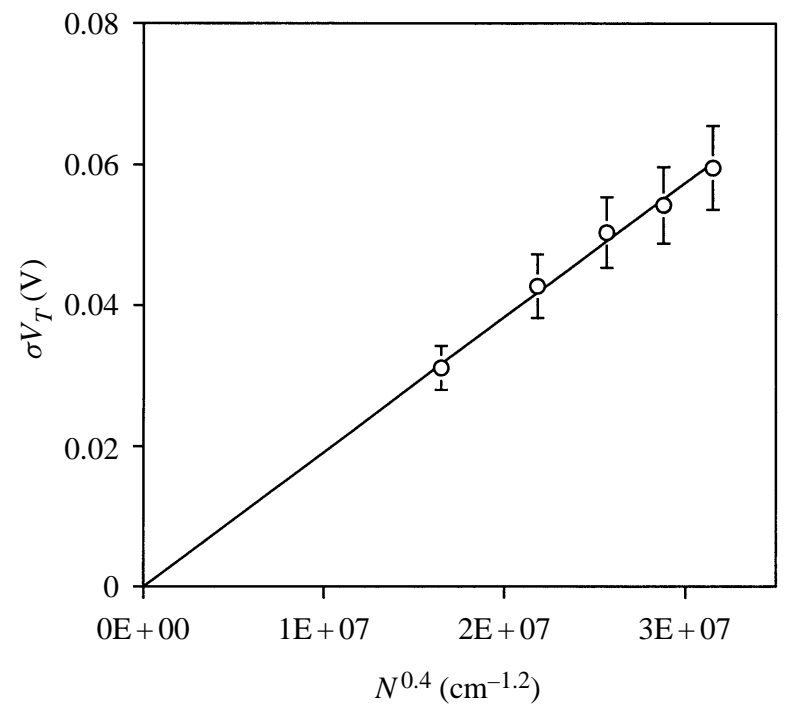

Fig. 3. Doping concentration dependence of $\sigma V_{T}$.

of the available analytical models (Fig. 3). This discrepancy is due to the fact that the analytical models take into account only the fluctuation in the total number of dopants in the channel depletion region but do not take into consideration the random position and clustering of the individual dopants.

\subsection{Fluctuation resistant MOSFET architectures}

The random dopant-induced parameter fluctuations can be substantially reduced in MOSFET architectures with epitaxial channels and delta-doping [18]. These architectures have additional benefits in terms of optimal threshold voltage control and improved mobility [19-22]. The undoped epitaxial layer thickness is restricted to approximately one-fifth of the effective channel length, due to short channel effects. The introduction of a $\delta$-doped layer below the edge of the epitaxial channel additionally provides an efficient short channel and threshold voltage control. As shown in Fig. 4, the introduction of a $10 \mathrm{~nm}$ epitaxial layer in a $50 \times 50 \mathrm{~nm}$ MOSFET reduces the random dopant-induced threshold voltage fluctuations almost five times.

When partially depleted, a delta-doped layer behind the epitaxial channel will act as a ground plane efficiently suppressing the short-channel effects. Above a certain thickness of the epitaxial layer a pronounced 'anomalous' decrease in the threshold voltage fluctuation is observed with increase of the delta-doping (Fig. 5). This behaviour is associated with the screening of the potential fluctuations by the holes in the partially depleted delta-doped layer. Due to this 'anomalous' behaviour, for a range of thicknesses of the epitaxial layer, transistors with delta-doping and relatively low level of doping behind the epi-layer may have threshold voltage fluctuation resistance comparable to that of transistors without delta-doping but with much higher level of doping behind the epitaxial layer.

\subsection{The effect of the polysilicon gate}

The random dopants and the depletion in the polysilicon gate have a detrimental effect on the MOSFET parameter fluctuations. The effects have been studied in conventional MOSFETs with uniform channel doping and on low doped epitaxial channel devices [23]. Figure 6 shows the potential distribution in a simulated 


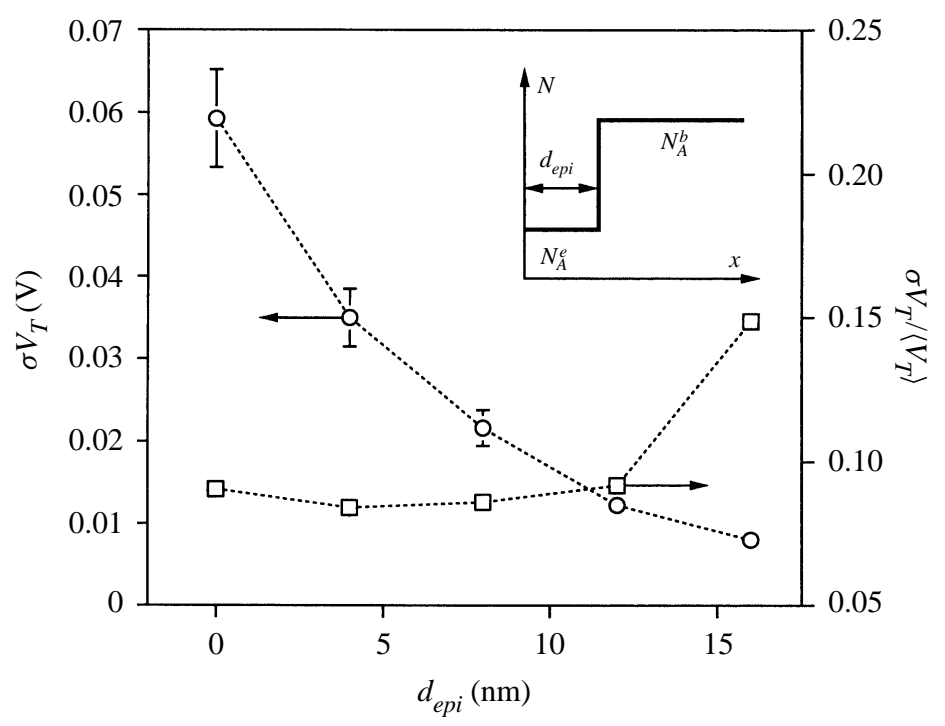

Fig. 4. Standard deviation of the threshold voltage, $\sigma V_{T}$, as a function of the thickness of the epitaxial layer $d_{\text {epi }}$.

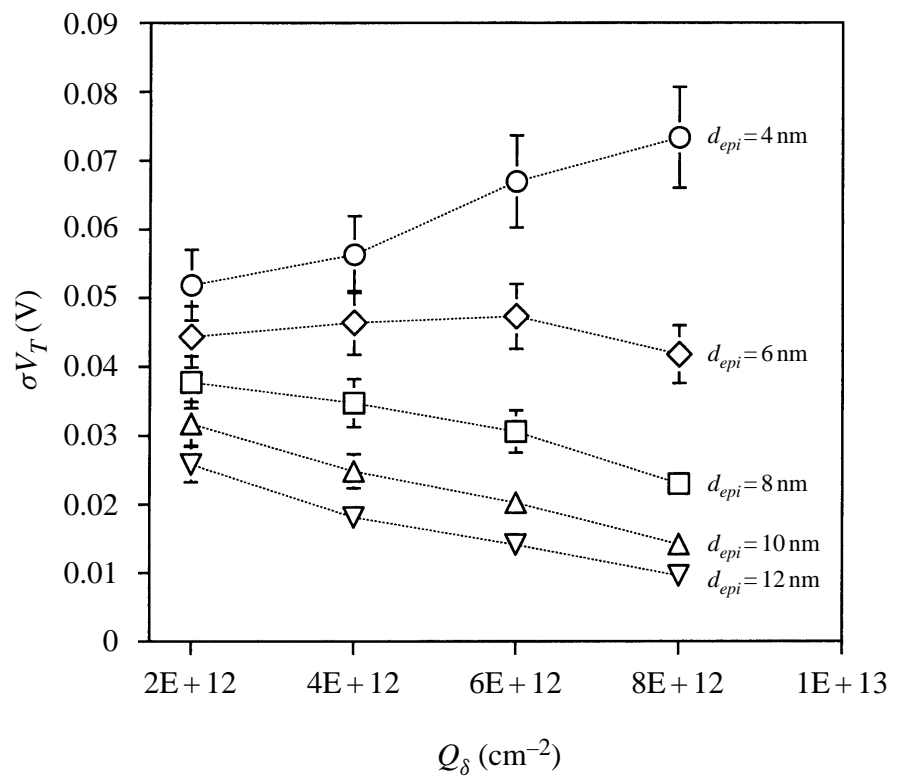

Fig. 5. Standard deviation in the threshold voltage $\sigma V_{T}$ as a function of the $\delta$-doping concentration $Q_{\delta}$ in epitaxial $\delta$-doped MOSFETs with various $d_{\text {epi }}$. Samples of 200 transistors.

MOSFET with the polysilicon gate 'flipped' up to show the potential fluctuations at the polysilicon-oxide interface. It has been shown that the polysilicon gate is responsible for a substantial fraction of the threshold voltage fluctuations in both type of devices when the gate oxide is scaled down to tunnelling thicknesses in the range $1-2 \mathrm{~nm}$.

The threshold voltage standard deviation as a function of the oxide thickness for a MOSFET with an $\mathrm{n}^{+}$ 


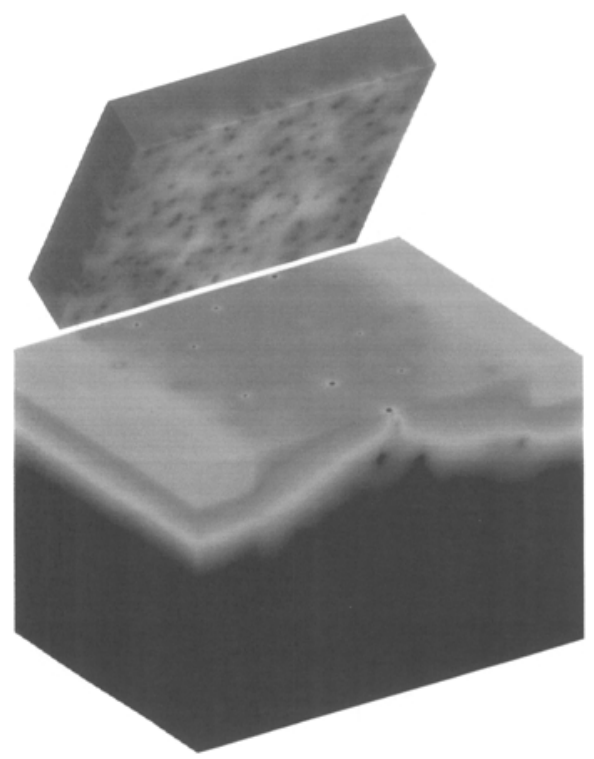

Fig. 6. 3D potential distribution corresponding to $V_{G}=V_{T}=0.723 \mathrm{~V}$ for a MOSFET with a polysilicon gate; channel doping concentration $N_{A}=5 \times 10^{18} \mathrm{~cm}^{-3}$, polysilicon doping concentration $N_{D}=5 \times 10^{19} \mathrm{~cm}^{-3}$, and $L_{e f f}=W_{e f f}=50 \mathrm{~nm}, x_{j}=7 \mathrm{~nm}$, $t_{o x}=3 \mathrm{~nm}$.

polysilicon gate has been compared with that of the metal-gate MOSFET. Both devices with uniform doping and epitaxial channels have been considered. For metal gate devices the fluctuations decrease linearly to zero with decreasing oxide thickness in agreement with most of the available analytical models. The polysilicon gate results are shifted up with respect to the metal gate results (Fig. 7). This shift results in a substantial percentage increase in $\sigma V_{T}$ when the gate oxide is scaled to its tunnelling limits in both uniform and epitaxial devices.

\subsection{Effect of single-electron interface trapping}

Our 3D 'atomistic' simulator is suitable for investigating the effect of single electron trapping at the interface on the channel conductivity in decanano MOSFETs [24]. Potential fluctuations generated by trapping/detrapping of single carriers at the interface may result not only in deterioration of the low-frequency noise performance in analogue circuits but also in local loss of functionality in memories and digital applications [25].

We use atomistic simulations at this stage to study only the effects associated with the local change in the carrier density due to the trapping of single electrons, assuming a constant mobility. The presented results are for decanano n-channel MOSFETs with a square geometry. Initially continuous doping is used in the simulations and only the trapped electrons in acceptor-type interface states are represented as individual discrete charges. A typical potential distribution for this case in a $50 \times 50 \mathrm{~nm}$ MOSFET with one electron trapped in the middle of the channel is shown in Fig. 8. Figure 9 represents the percentage variation in the current associated with the trapping as a function of the gate voltage in square MOSFETs ranging from $100 \times 100 \mathrm{~nm}^{2}$ down to $30 \times 30 \mathrm{~nm}^{2}$. The change is large in the subthreshold region and rapidly decreases above threshold where the charge of the trapped electron is screened by the mobile carriers charge in the channel.

When the 'atomistic' nature of such small-scale devices is taken into account, the effect of a single trapped 


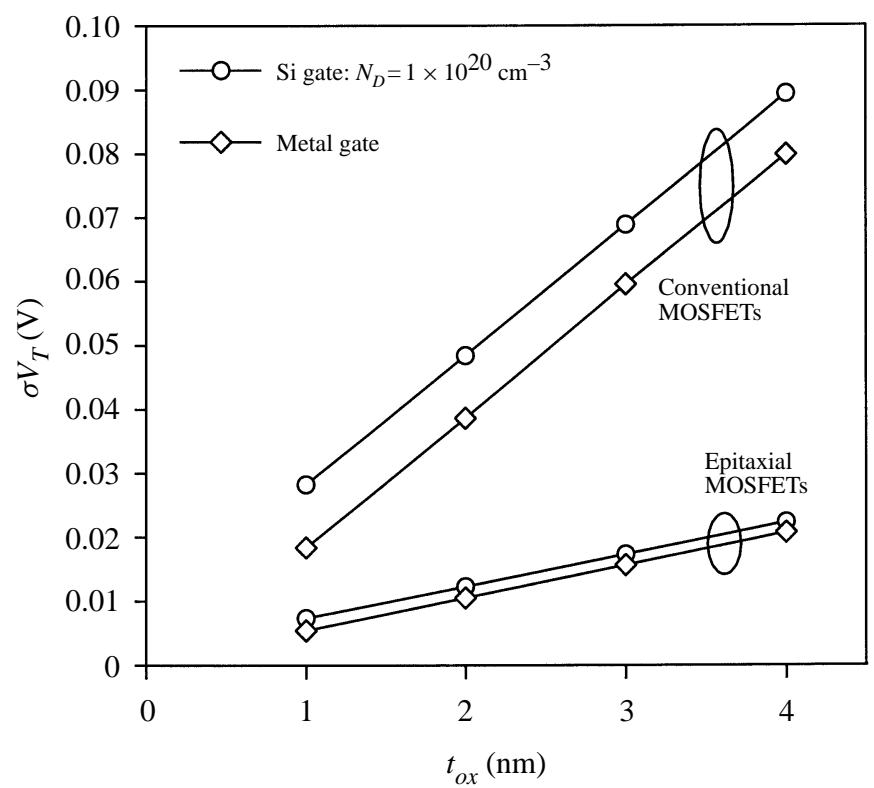

Fig. 7. Dependence of the threshold voltage standard deviation $\sigma V_{T}$ on the oxide thickness $t_{o x}$ for polysilicon and metal gate MOSFETs with $L_{e f f}=W_{e f f}=50 \mathrm{~nm}, x_{j}=7 \mathrm{~nm}, N_{A}=5 \times 10^{18} \mathrm{~cm}^{-3}$. The polysilicon doping concentration is $N_{D}=1 \times 10^{20} \mathrm{~cm}^{-3}$. Results for conventional and epitaxial MOSFETs are shown.

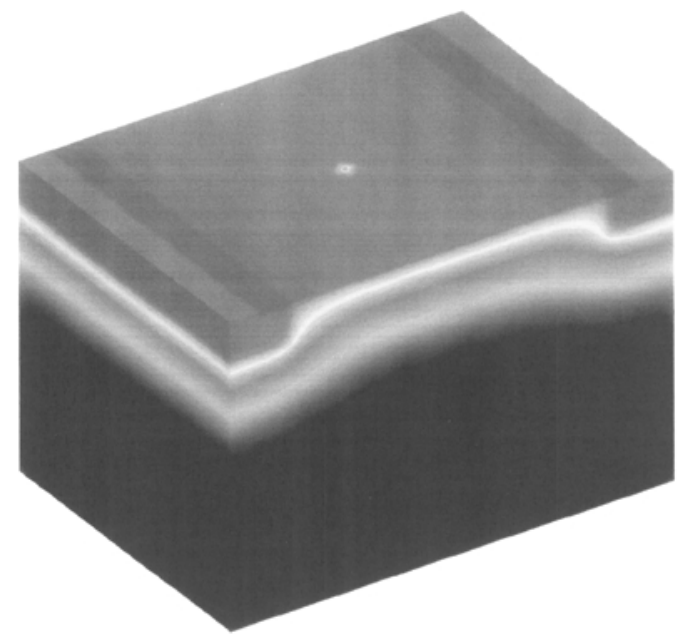

Fig. 8. 3D potential distribution with one electron trapped in the middle of the channel in a $50 \times 50 \mathrm{~nm}^{2}$ MOSFET

electron can be more dramatic. The current density throughout the channel varies as the current percolates between the potential barriers produced by the discrete dopant charges. This results in a number of current paths from source to drain. If an electron should become trapped at some point along one of these current paths, the resultant fixed charge introduced could effectively block that particular path, resulting in a significant drop in the current. An experiment where a device with random dopants was simulated 100 times, each 


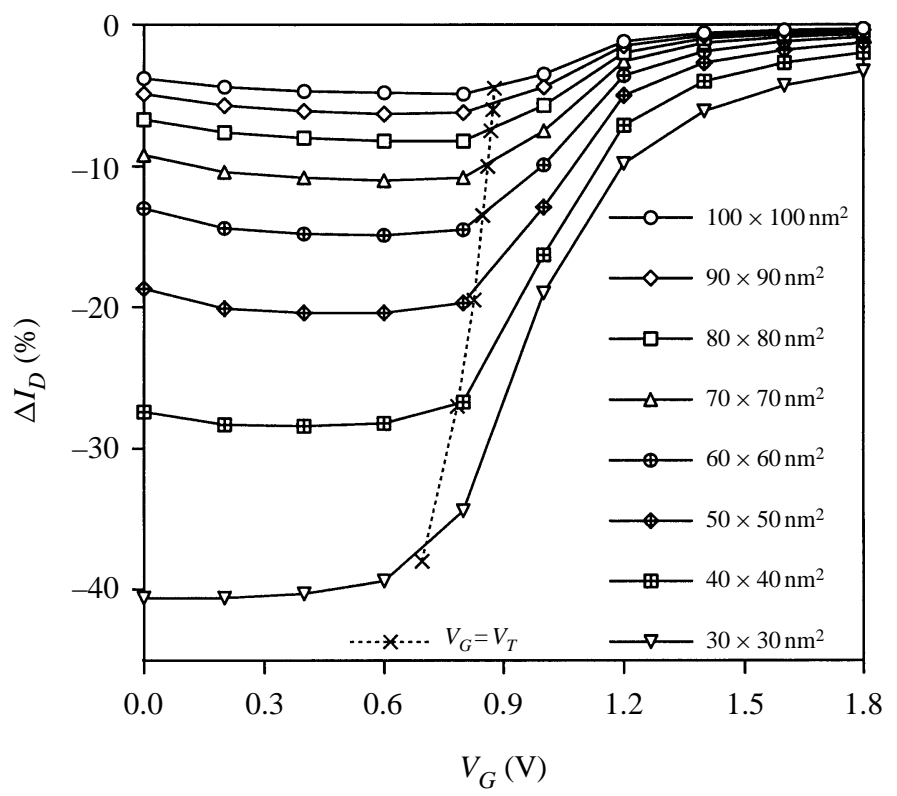

Fig. 9. Percentage change in the current resulting from the trapping of one electron in the middle of the channel of a square MOSFET.

time with an electron trapped in a random location within the channel, demonstrated a further reduction in current, up to $40 \%$, when the electron was trapped in a 'worst-case' location.

\section{Quantum corrections in the 'atomistic' simulations}

\subsection{Simulation approach}

The increase in doping concentration and reduction in oxide thickness in MOSFETs scaled to sub-100 nm dimensions results in a strong quantization in the inversion layer, with a corresponding increase in the threshold voltage [26]. However, all previous 3D simulation studies of random dopant fluctuation effects [11, 15, 20] do not take into account quantum effects. It is important to evaluate to what extent the quantum effects would affect the random dopant-induced threshold voltage fluctuation and lowering, and to what degree the threshold voltage lowering may compensate for the increase in the threshold voltage associated with inversion layer quantization [27].

The rigorous approach for modelling of inversion layer quantisation effects consists of the coupled solution of the Schrödinger and Poisson equations [26,28]. We, however, use a 3D implementation of the density-gradient (DG) model developed in [29] to introduce quantum corrections in the drift-diffusion simulations. This is an approximate approach for introducing quantum mechanical corrections into a macroscopic transport description by considering a more general equation of state for the electron gas, depending on the density gradient.

The major reason for choosing the DG method is that it represents an extension to the drift-diffusion model which can account for at least some of the quantum mechanical effects (e.g. quantum smoothing of the carrier density profiles, quantum confinement and tunnelling), taking advantage of the full set of state-ofthe-art numerical computational methods developed for solving the classical DD equation.

At low drain voltage we solve self-consistently the 3D Poisson equation for the potential $\psi$ and the 3D 


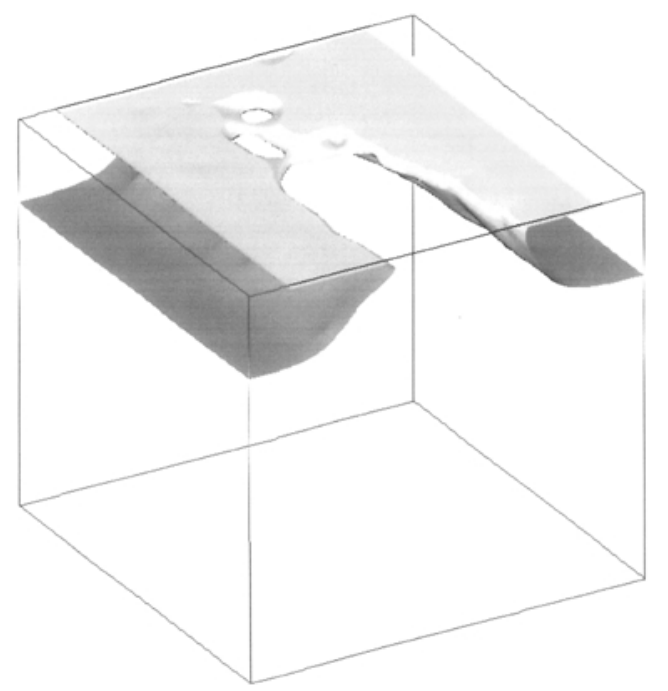

Fig. 10. One equi-concentration contour corresponding to the potential distribution in Fig. 1. The inversion charge distribution peaks below the $\mathrm{Si} / \mathrm{SiO}_{2}$ interface.

DG approximation of the Schrödinger equation (1):

$$
2 b_{n} \frac{\nabla^{2} \sqrt{n}}{\sqrt{n}}=\phi_{n}-\psi+\frac{k T}{q} \ln \frac{n}{n_{i}},
$$

where a microscopic expression of the macroscopic factor $b_{n}$ has been derived in [30] which reads $b_{n}=$ $\hbar^{2} /\left(12 q m_{n}^{*}\right), \phi_{n}$ is the generalized quasi-Fermi potential, and all other symbols have the conventional meaning. The right-hand side of (1) represents the Boltzmann statistics for electrons and the left-hand side can be interpreted as a quantum mechanical correction to the Boltzmann statistics.

The current at low drain voltage is extracted from the resistance of the MOSFET calculated from the electron concentration distribution as described in [15]. The potential distribution obtained from the selfconsistent solution of the Poisson equation and (1) is illustrated in Fig. 1 at gate voltage equal to the threshold voltage. Strong potential fluctuations at the $\mathrm{Si} / \mathrm{SiO}_{2}$ interface associated with the discrete dopants can still be observed. One electron equi-concentration contour which corresponds to this solution is presented in Fig. 10. The equi-concentration contour highlights the basic features of the quantum charge distribution. The quantum confinement in the channel results in a smoothing of the carrier density profile with a maximum in the electron concentration, located approximately $1.5 \mathrm{~nm}$ below the interface. As can be seen from Fig. 10, the 3D solution of (1) also contains the effects of the lateral confinement which in turn results in narrowing of the current channels percolating through the 'valleys' in the fluctuating surface potential. We also believe that the penetration of the solution through sharp potential barriers associated with individual dopants represent tunneling effects [31].

Since (1) is only an approximation to the Schrödinger equation, the DG model has to be validated against a full self-consistent solution of the Poisson-Schrödinger equation. This is a difficult task in 3D, particularly in a complex solution domain representing a MOSFET, and potential incorporating fluctuations from discrete dopants. Therefore we validate the DG approach against full band Poisson-Schrödinger simulations [26] only in the 1D case and for continuous doping. Our DG results for the quantum mechanical threshold voltage shift, $V_{T}(Q M)-V_{T}$ (Classical), shown in Fig. 11, using the value of electron effective mass, $m^{*}=0.19 m_{0}$, as recommended in [32], are in excellent agreement with the shift reported in [26]. The range of doping concentration used in the comparison corresponds to that of properly scaled MOSFETs with channel lengths 


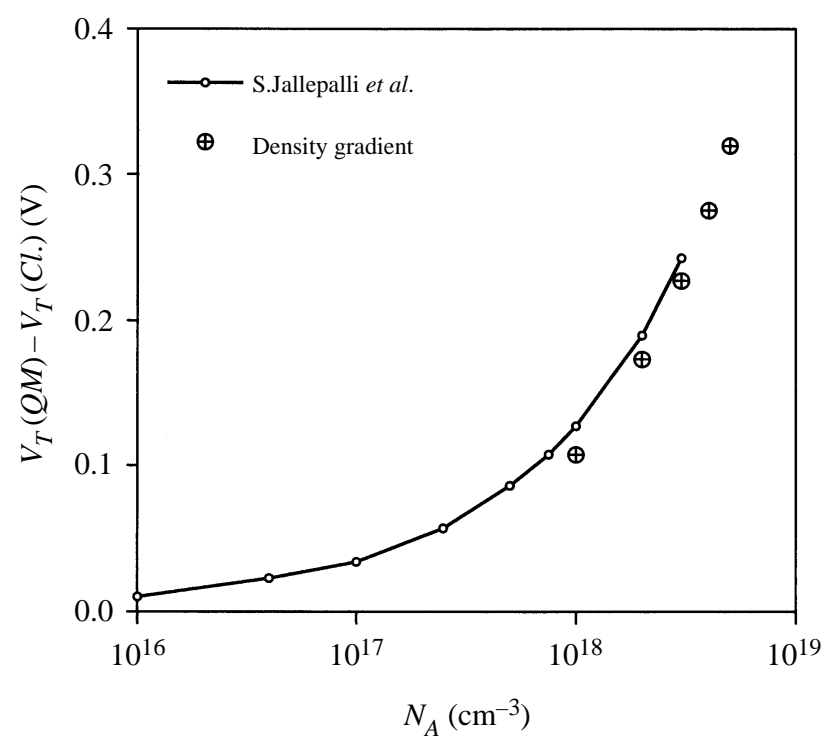

Fig. 11. Quantum mechanical threshold voltage shift as a function of the doping concentration. A comparison between DG and full band Poisson-Schrödinger results [26] for continuous doping distribution.

below $100 \mathrm{~nm}$. As has been pointed out in [33], this electron mass seems to represent well the quantum potential correction in cases when straightforward comparison with experiment is possible. However, we believe that the effective mass has to be treated as an adjustable parameter in the DG approach.

\subsection{Results and discussion}

The 'atomistically' simulated threshold voltage standard deviation $\sigma V_{T}$ for a set of standard MOSFETs with different oxide thicknesses with metal and polysilicon gates is compared in Fig. 12. The devices have uniform doping concentration $N_{A}=5 \times 10^{18} \mathrm{~cm}^{-3}$, junction depth $x_{j}=7 \mathrm{~nm}$, equal channel length and width $L_{e f f}=W_{e f f}=50 \mathrm{~nm}$, and polysilicon doping concentration $N_{D}=1 \times 10^{20} \mathrm{~cm}^{-3}$. Both results from classical 'atomistic' simulation and simulations including DG correction for the quantum mechanical effects are presented in the same figure. The quantum mechanical calculated standard deviation exhibits the same linear dependence on the oxide thickness as the one resulting from the classical 'atomistic' simulations but is shifted up and does not scale to zero with the oxide thickness.

Quantum confinement can enhance the fluctuations by $50 \%$ if the oxide thickness is below $1.5 \mathrm{~nm}$, as expected near the end of the Roadmap [34]. The effect is further enhanced by the polysilicon gate due to the superposition of the fluctuations generated by the polysilicon doping impurities and the ones originating from the silicon substrate, and the effective increase in the oxide thickness due to the poly-depletion effect.

\section{Conclusions}

A statistical 3D atomistic simulation approach, consisting of the simulation of hundreds of microscopically different devices, has been adopted to investigate the effects associated with the random impurity distribution in decanano MOSFETs. The random dopant induced potential fluctuations result in threshold voltage variations and reduction in the average threshold voltage with respect to the continuous doping case. Fluctuationresistant decanano MOSFET architectures including epitaxial channels and delta doping have been studied 


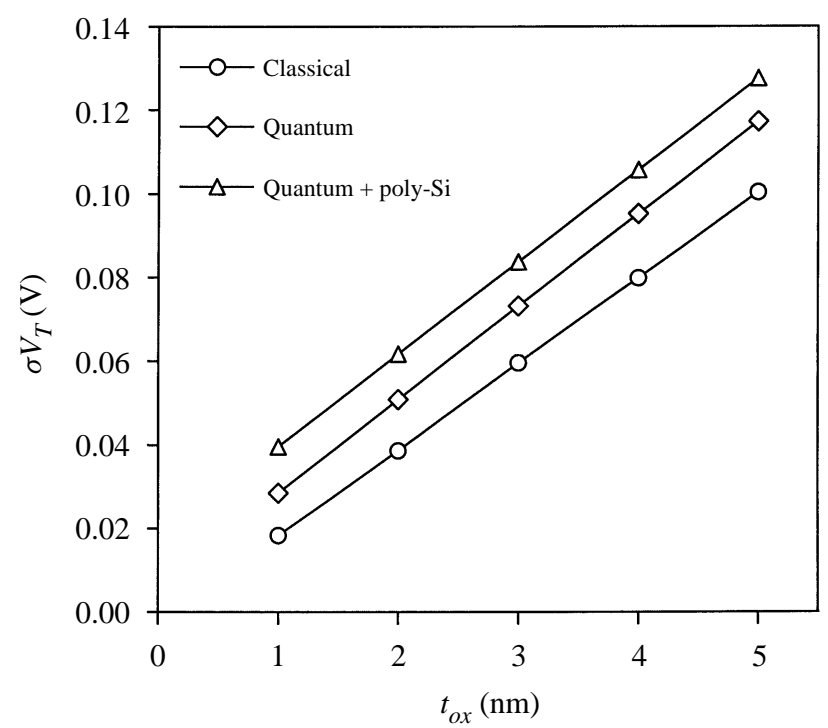

Fig. 12. Standard deviation in threshold voltage $\sigma V_{T}$ as a function of oxide thickness with metal and polysilicon gates. Results from classical atomistic simulations and simulations including DG correction for QM effects are shown.

at the atomistic level. Efficient suppression in the threshold voltage fluctuations has been observed in devices with low-doped epitaxial channels. For certain thicknesses of the epitaxial layer the introduction of $\delta$-doping results in reduction in the fluctuations, due to screening in the delta doping layer, offering new means for charge fluctuation control. The effect of random dopants in the polysilicon gate on the threshold voltage fluctuations has been investigated. A substantial increase in the fluctuations has been reported for thin oxide devices associated with both polysilicon depletion effects and random dopants in the gate.

The effect of single-charge trapping/detrapping at the interface has also been studied in our atomistic simulation framework. The trapping of a single charge can have a significant impact on the channel current in narrow decanano MOSFETs, particularly in the subthreshold region. This can be even more dramatic when the atomistic nature of the channel doping is considered.

It has been demonstrated that accounting for the quantum effects in 'atomistic' simulations results in an increase in the threshold voltage fluctuations. The quantum enhancement in the threshold voltage uncertainty amounts to more than $50 \%$ in MOSFETs with oxide thicknesses below $1.5 \mathrm{~nm}$, expected near the end of the Roadmap.

Acknowledgement-This work is supported by NASA Ames Research Center grant NAG 2-1241.

\section{References}

[1] R. W. Keys, Physical limits in digital electronics, Proc. IEEE 63, 740 (1975).

[2] B. Hoeneisen and C. A. Mad, Fundamental limitations in microelectronics-I, MOS technology, SolidState Electron. 15, 819 (1972).

[3] T. Hagigava, K. Yamaguchi, and S. Asai, Proceedings of the 1982 Symposium VLSI Technol., Digest Technical Papers, p. 46.

[4] K. R. Lakshmikumar, R. A. Hadaway, and M. A. Copeland, Characterisation and modelling of mismatch in MOS transistors for precision analogue design, IEEE J. Solid State Circuits SC-21, 1057 (1986). 
[5] M. Steyaert, J. Bastos, R. Roovers, P. Kinget, W. Sansen, B. Graindourse, A. Pergot, and Er. Janssens, Threshold voltage mismatch in short-channel MOS transistors, Electron. Lett. 30, 1546 (1994).

[6] T. Mizuno, M. Iwase, H. Niiyama, T. Shibata, K. Fujisaki, T. Nakasugi, A. Toriumi, and U. Ushiku, Proceedings of the 1994 VLSI Symposium, p. 13.

[7] T. Mizuno, J. Okamura, and A. Toriumi, Experimental study of threshold voltage fluctuation due to statistical variation of channel dopant number in MOSFETs, IEEE Trans. Electron Devices 41, 2216 (1994).

[8] O. R. dit Buisson and G. Morin, Proceedings of ESSDERC'96, edited by G. Bakarani and M. Rudan (1996) p. 731.

[9] M. Eisele, I. Bertold, R. Thewes, F. Wohlrab, D. Schmitt-Landsidel, and W. Weber, Proceedings of IEDM'95 Dig. Tech. Papers, p. 67.

[10] J. T. Horstmann, U. Hilleringmann, and K. F. Goser, Matching analysis of deposition defined 50-nm MOSFETs, IEEE Trans. Electron Devices 45, 299 (1997).

[11] H.-S. Wong and Y. Taur, Proceedings of the IEDM'93, Technical Digest Papers, (1993) p. 705.

[12] T. Mikolajick and H. Ryssel, Influence of statistical dopant fluctuations on MOS transistord with deep submicron channels, Microelectron. Eng. 21, 419 (1993).

[13] J.-R. Zhou and D. K. Ferry, Proceedings of the 3rd International Workshop on Computational Electronics, Portland, OR, 1994 (Plenum Press, New York), p. 74.

[14] D. Vasileska, W. J. Gross, and D. K. Ferry, Modeling of deep-submicrometer MOSFETs: random impurity effects, threshold voltage shifts and gate capacitance attenuation, Extended Abstracts IWCE-6, Osaka 1998, IEEE Cat. No. 98EX116, p. 259.

[15] A. Asenov, Random dopant induced threshold voltage lowering and fluctuations in sub $0.1 \mu \mathrm{m}$ MOSFETs: a 3-D 'atomistic' simulation study, IEEE Trans. Electron Devices 45, 2505 (1998).

[16] H. K. Gummel, A self-consistent iterative scheme for one-dimensional steady state transistor calculations, IEEE Trans. Electron Devices 11, 455 (1964).

[17] A. Asenov, A. R. Brown, J. H. Davies, and S. Saini, Hierarchical approach to 'atomistic' 3D MOSFET simulation, IEEE Trans. Comput. Aided Des. 18, 1558 (1999).

[18] A. Asenov and S. Saini, Supression of random dopant induced threshold voltage fluctuations in sub$0.1 \mu \mathrm{m}$ MOSFETs with epitaxial and delta doped channels, IEEE Trans. Electron Devices 46, 1718 (1999).

[19] K. Nishiohara, N. Shiguo, and T. Wada, Effects of mesoscopic fluctuations in dopant distributions on MOSFET threshold voltage, IEEE Trans. Electron Devices 39, 634 (1992).

[20] P. A. Stolk, F. P. Widdershoven, and D. B. M. Klaassen, Modeling statistical dopant fluctuations in MOS transostors, IEEE Trans. Electron Devices 45, 1960 (1998).

[21] K. Takeuchi, T. Tatsumi, and A. Furukawa, Proceedings of the IEDM'97, Technical Digest Papers.

[22] K. Noda, T. Tatsumi, T. Uchida, K. Nakajima, H. Miyamoto, and C. Hu, A 0.1- $\mu$ m delta doped MOSFET fabricated with post-low-energy implanting selective epitaxy, IEEE Trans. Electron Devices $\mathbf{4 5}$, 809 (1998).

[23] A. Asenov and S. Saini, Polysilicon gate enhancement of the random dopant induced threshold voltage fluctuations in sub $100 \mathrm{~nm}$ MOSFETs with tunnelling oxide, IEEE Trans. Electron Devices, in press.

[24] R. Balasubramaniam, A. Asenov, J. H. Davies, and A. R. Brown, Effect of single electron interface trapping in decanano MOSFETs: a 3D 'atomistic' simulation study, presented at SIMD'99, Maui, HI, December 1999, Superlatt. Microstruct., doi:10.1006/spmi.2000.0823.

[25] Y. Shi, H. M. Bu, X. L. Yuan, and Y. D. Zheng, Random telegraph signals in very narrow channel MOSFET, workshop abstracts, 1999 Silicon Nanoelectronics Workshop, Kyoto, p. 28.

[26] S. Jallepalli, J. Bude, W.-K. Shih, M. R. Pinto, C. M. Maziar, and A. F. Tasch, Jr., Electron and hole quantisation and their impact on deep submicron silicon p- and n-MOSFET characteristics, IEEE Trans. Electron Devices 44, 297 (1997). 
[27] A. Asenov, G. Slavcheva, A. R. Brown, J. H. Davies, and S. Saini, Quantum Mechanical Enhancement of the Random Dopant Induced Threshold Voltage Fluctuations and Lowering in Sub 0.1 micron MOSFETs, presented at IEDM'99 in Proceedings of the IEDM'99, Technical Digest Papers.

[28] K. S. Krisch, J. D. Bude, and L. Manchanda, Gate capacitance attenuation in MOS devices with thin gate oxides, IEEE Electron Device Lett. 17, 512 (1996).

[29] M. G. Ancona and H. F. Tiersten, Microscopic physics of the silicon inversion layer, Phys. Rev. B35, 7959 (1987).

[30] M. G. Ancona and G. I. Iafrate, Quantum correction to the equation of state of an electron gas in a semiconductor, Phys. Rev. B39, 9536 (1989).

[31] M. G. Ancona, Z. Yu, R. W. Dutton, P. J. V. Voorde, and M. Cao, Proceedings of the SISPAD'99, Kyoto.

[32] C. S. Rafferty, B. Biegel, Z. Yu, M. G. Ancona, J. Bude, and R. W. Dutton, Proceedings of the SISPAD'98, edited by K. De Meyer and S. Biesemans, p. 137.

[33] B. A. Biegel, C. S. Rafferty, Z. Yu, M. G. Ancona, and R. W. Dutton, Proceedings of the Gigascale Integration Technology Symposium, 35th Annual Technical Meeting, 1998 (Society of Engineering Science (SES35)), Sept. 27-30, Pullman, USA, WA, p. 53.

[34] National Technology Roadmap for Semiconductors, 1997 Edition, Semiconductor Industry Association. 